\title{
Lymphocytic infiltration in helicobacter pylori gastritis
}

\author{
Fatma A. Ali ${ }^{a}$, Sheren F. Mahmoud ${ }^{\text {b }}$, Ali A. Said ${ }^{c}$, Eman M. Salah Elden ${ }^{\text {b }}$ \\ ${ }^{a}$ Pathology Department, Faculty of Medicine,South Valley University, Qena, Egypt. \\ ${ }^{\mathrm{b}}$ Pathology Department, Faculty of Medicine ,Sohage University, Sohage,Egypt. \\ ${ }^{\mathrm{c}}$ Gastroenterology and Tropical Medicine Department, Qena University Hospital,SouthVally \\ university.
}

\begin{abstract}
:
Background: Severity of lymphocytic infiltration to gastric mucosa is a good indicator for severity of H. Pylori gastritis

Objectives: To evaluate the lymphocytic infiltration in the gastric mucosa associated with $\mathrm{H}$. Pylori infection.

Patients and Method(s): This study done on 50 cases with chronic gastritis and H.Pylori were detected by using geimsa stain with evaluation of degree of lymphocytic infiltration

Results: H. Pylori infection was detected in $86 \%$ of cases with mild, moderate and severs infection in $(34 \%, 42 \%$ and $10 \%)$ respectively. The lymphocytic infiltration found in $66 \%$ of cases with four levels (mild 24\%, moderate 16\%, sever without L.F 18\%, sever with L.F 8\%). There was statistically significant relation between severity of H.Pylori infection and severity of lymphocytic infiltration $(\mathrm{p} \leq 0.001)$.
\end{abstract}

Conclusion: Lymphocytic infiltration is an important feature of H.Pylori gastritis.

Keywords: H.Pylori, Chronic gastritis, Lymphocytic infiltration.

\section{Introduction:}

Helicobacter pylori previously named Campylobacter pyloridis, is a Gram-negative, microaerophilic bacterium found in the stomach. It was present in patients with chronic gastritis and gastric ulcers, conditions that were not previously believed to have a microbial cause. It is also linked to the development of duodenal ulcers and stomach cancer. However, over 80 percent of individuals infected with the bacterium are asymptomatic and it has been postulated that it may play an important role in the natural stomach ecology (Blaser, 2006).

A characteristic feature of the chronic gastritis induced by H.Pylori is the presence of mucosaassociated lymphoid tissue (MALT), which consists of lymphoid aggregates and organized follicles ( Mazzella et al., 1999).

The lamina propria of stomach is a loose connective tissue that contains a few numbers of lymphocytes and plasma cells. Presence of

lymphoid follicles denoting previous or recent H.Pylori infection (Owen et al., 2007). Chronic gastritis can be classified according to the presence or absence of $\mathrm{H}$. Pylori into $\mathrm{H}$. pyloriassociated and non-H. Pylori-associated forms (Marshall et al., 1994). There is no doubt that H. Pylori infection is responsible for the majority of cases of gastritis. In general, the inflammation induced by this organism is a chronic active gastritis which means that both lymphocytes and neutrophiles infiltrate the mucosa in a characteristic manner (Weck et al., 2009). Measuring of the severity of the inflammatory lesions occur by grades range from 0 ; absence of inflammatory cells in any of the specimens to 4 , according to ubdated Sydney classification (Taha et al., 2016). Gastritis due to H. Pylori is caused by a complicated interaction among a variety of T cells (Nader et al., 2016). 


\section{Patients and methods:}

This prospective study was performed on fifty patients suffering from upper gastrointestinal upsets admitted to the department of tropical medicine and gastroenterology in Qena University hospital for upper endoscopic evaluation from January 2018 to June of the same year. Tissue biopsies were obtained from the stomach for histopathological diagnosis in the histopathology lab of the same hospital. Biopsies were randomly taken from the endoscopically suspected areas and put in only one patient-labeled container without separation of fundic, cardiac or pyloric samples.

All clinical data including patient age, sex, complaint, site of gastric biopsy and endoscopic findings were obtained from the clinical referral sheets accompanying biopsy specimens.

The main complaints for patients were dyspepsia, epigastric pain, heart burn and repeated vomiting in ten, sixteen, twelve and eight patients respectively. Three patients presented with hematemesis and only one suffered from anemia of unknown cause.

From each biopsy, formalin-fixed paraffinembedded blocks were prepared and two sections obtained from each block to be stained by H\&E, Giemsa stain.

\section{Results:}

We evaluated 50 cases, 25 males (50\%) and 25 females $(50 \%)$. Their ages ranged from 17 to 82 years old with a mean age of $42.42 \pm 17.5$ and a median is 38.5 years old. Helicobacter pylori infection was detected in 43 out of 50 patients $(86 \%)$. The severity of infection was mild, moderate and severe in (17, 21 and 5) cases respectively with percentage of $(34 \%, 42 \%$, $10 \%$ ) according to Sydney classification. Table and Figure 1.
Table 1. Distribution of studied population according to severity of helicobacter pylori.

\begin{tabular}{|lllc|}
\hline $\begin{array}{l}\text { H. } \\
\text { pylo } \\
\text { ri }\end{array}$ & Negative & $\mathbf{7}$ & $\mathbf{1 4 \%}$ \\
& Mild & 17 & $34 \%$ \\
& Moderate & 21 & $42 \%$ \\
\hline & Sever & 5 & $10 \%$ \\
\hline
\end{tabular}

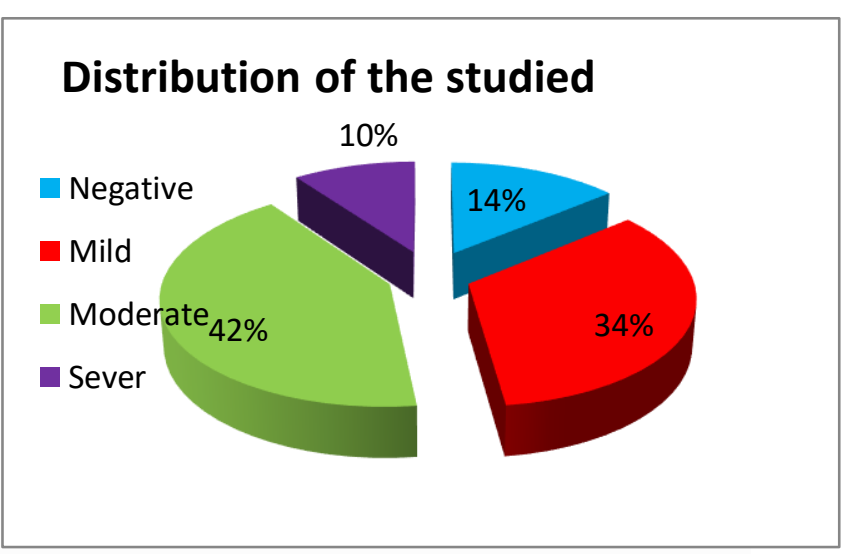

Figure 1. Distribution of studied population according to severity of helicobacter pylori.

Chronic inflammatory cell infiltrate was detected in thirty three of cases, but with variable intensities (mild, moderate, sever without lymphoid follicles formation infiltration and sever with lymphoid follicles formation) in $(12,8,4,9)$ respectively with percentages of $(24 \%, 16 \%, 8 \%, 18 \%)$ respectively. Figure 2. 


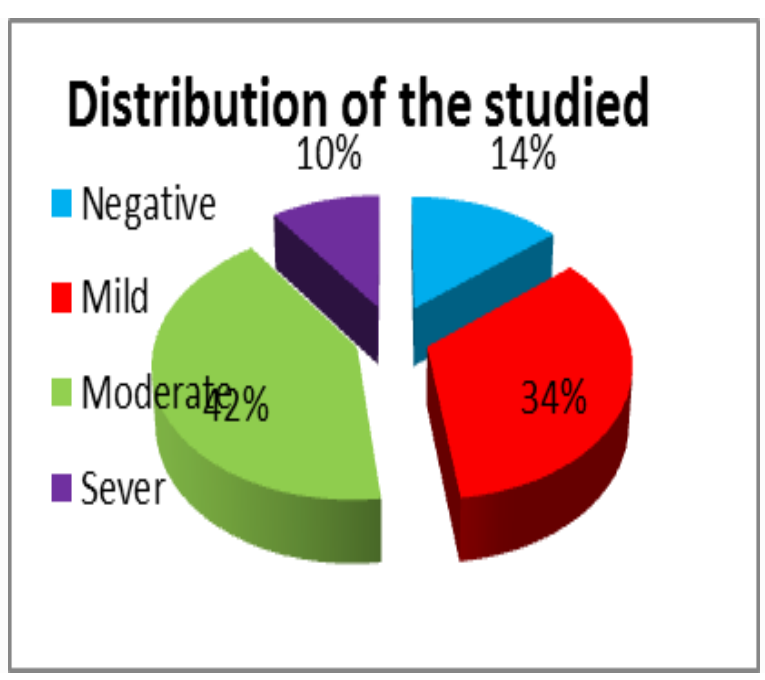

Figure 2. Distribution of studied population according to lymphocytic infiltration to gastric mucosa.

The relation between severity of lymphocytic infiltration and severity of H.pylori infection was significant ( $\mathrm{p}$ value $<\mathbf{0 . 0 0 1}$ ) as shown in Figure 3.

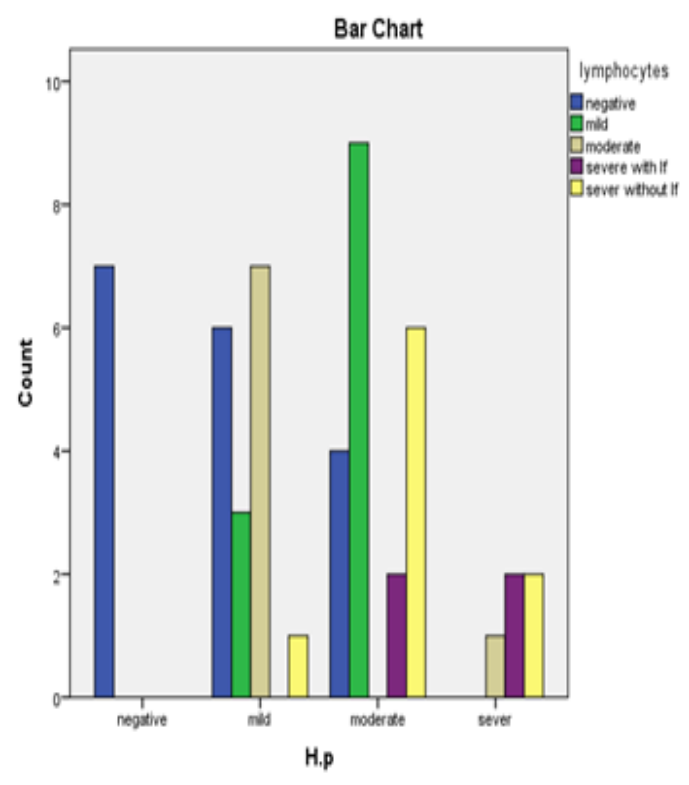

Figure 3. The relation between severity of helicobacter pylori and severity of lymphocytic infiltration to gastric mucosa.
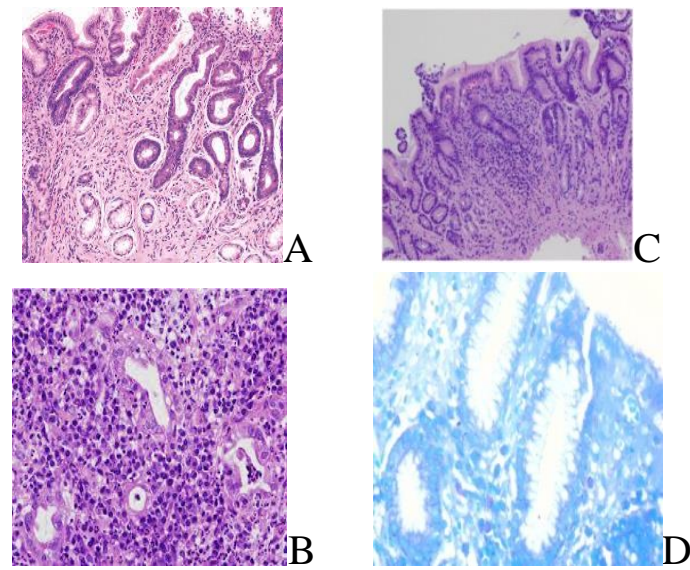

Figure 4.

A: moderate lymphocytic infiltration.

B: Severe lymphocytic infiltration without lymphoid follicles formation.

C: Severe lymphocytic infiltration with lymphoid follicles formation.

D:H.Pylori bacilli by gimsa stain seen at the lumen of the glands.

\section{Discussion:}

In our study, the age range of the 50 studied patients (with gastritis) was: 25-59 years, the mean age was $42.4 \pm 17$ years, and the median age was 38.5 years. $25 / 50(50 \%)$ of cases were males and $25 / 50(50 \%)$ were females with male to female ratio was $1: 1$.

Other study also confirmed that age and sex are non-significant in H.Pylori gastritis only socioeconomic level plays a role (Shiferaw et al., 2019).

In our study 50 cases 17 cases were negative for lymphocytic infiltration (34\%), thirty three of cases were positive $(66 \%)$ but with a variable intensities (mild, moderate, sever without lymphoid follicles formation and sever with lymphocytic infiltration) in $(12,8,9,4)$ cases respectively. The percentages were $(24 \%, 16 \%, 18 \%, 8 \%)$ respectively.

Very close data was obtained from another study among the 100 cases, 79 patients (79\%) showed evidence of MALT in at least one biopsy specimen taken from the stomach and 21 cases (21\%) had no evidence of MALT. H. pylori infection was detected in $74(74 \%)$ patients. Lymphoid follicles were detected more frequently in H. Pylori-positive patients (59\%) 
compared to H. Pylori-negative cases (3\%) ( $\mathrm{P}<0.001)$ (Bashiri et al., 2019).

That explained by another results that suggest the interaction between CCL20 and CCR6 may play a role in chemokine-mediated lymphocyte trafficking during gastric inflammation in $H$. pylori infection. (Morrison et al., 2007).

\section{Conclusion:}

Lymphocytic infiltration is an important feature of H.Pylori gastritis. Over infiltration by lymphocytes and lymphoid follicles formation occur in cases of severe infection by H.Pylori.

\section{References:}

Blaser M. J, (2006). Who are we? Indigenous microbes and the ecology of human diseases.EMBO Reports; 7 (10): 956-60.

Mazzucchelli L, Andrea B, Andreas K, Patrik S, Jean A, Laissue, Marco B and Mariagrazia U, (1998). MALT associated gastritis.GASTROENTEROLOGY;(114):482-92

Owen D. A, (2007). Stomach. Histology for pathologists, Philadelphia;(3):589-602.

Marshall BJ, Royce $\mathbf{H}$ and Annear D. I, (1994). Original isolation of Campylobacter pyloridis from human gastric mucosa. FEMS Microbial Lett ;(25):83-85.

Weck M, Gao $L$ and Brenner H, (2009). Helicobacter pylori infection and chronic atrophic gastritis. Epidemiology; 20(4):569-74.

Taha H, Samia I, Ibrahim H,Fadi I and Malek G (2016). Helicobacter pylori chronic gastritis updated Sydney grading in relation to endoscopic findings. Journal of Microscopy and Ultrastructure ; 4(99):8-10.

Nader B, Fatemeh D and Ghorbanali R, (2016). Role of Regulatory T-cells in Different Clinical Expressions of Helicobacter pylori Infection. Gastroenterology; 47 (4):245-254.

Shiferaw G and Abera D, (2019). Magnitude of Helicobacter pylori and associated risk factors among symptomatic patients. BMC infectious Disease;19:118-120.
Bashiri H, Esmaeil A, Vossoughinia H, Ghaffarzadegan K, Raziei H, and Bozorgomid A, (2019). Association Between Gastric Lymphoid Follicles (Precursor Of MALT Lymphomas) And H. pylori Infection At A Referral Hospital In Iran. Clinical and Expremental Gastroentrology; (4)12: 409-413. 
